\title{
A RESULT CONCERNING TWO-SIDED CENTRALIZERS ON ALGEBRAS WITH INVOLUTION
}

\author{
NEJC ŠIROVNIK AND JOSO VUKMAN
}

Abstract. The purpose of this paper is to prove the following result. Let $X$ be a complex Hilbert space, let $\mathscr{L}(X)$ be the algebra of all bounded linear operators on $X$ and let $\mathscr{A}(X) \subset \mathscr{L}(X)$ be a standard operator algebra, which is closed under the adjoint operation. Let $T: \mathscr{A}(X) \rightarrow \mathscr{L}(X)$ be a linear mapping satisfying the relation $3 T\left(A A^{*} A\right)=T(A) A^{*} A+A T\left(A^{*}\right) A+A A^{*} T(A)$ for all $A \in \mathscr{A}(X)$. In this case $T$ is of the form $T(A)=\lambda A$ for all $A \in \mathscr{A}(X)$, where $\lambda$ is some fixed complex number.

Mathematics subject classification (2010): 16N60, 46B99, 39B42.

Keywords and phrases: Ring, ring with involution, prime ring, semiprime ring, Banach space, Hilbert space, standard operator algebra, $H^{*}-$ algebra left (right) centralizer, two-sided centralizer.

\section{REFERENCES}

[1] W. Ambrose, Structure theorems for a special class of Banach algebras, Trans. Amer. Math. Soc. 57 (1945), 364-386.

[2] K. I. Beidar, W. S. Martindale 3RD, A. V. Mikhalev, Rings with generalized identities. Marcel Dekker, Inc., New York, (1996).

[3] D. Benkovič, D. Eremita, Characterizing left centralizers by their action on a polynomial, Publ. Math. (Debr.) 64 (2004), 343-351.

[4] D. Benkovič, D. ERemita, J. Vukman, A characterization of the centroid of a prime ring, Studia Sci. Math. Hungar. 45, 3 (2008), 379-394.

[5] M. BREŠAR, Jordan mappings of semiprime rings, J. Algebra 127 (1989), 218-228.

[6] M. Fošner, J. Vukman, An equation related to two-sided centralizers in prime rings, Houston J. Math. 35, 2 (2009), 353-361.

[7] I. Kosi-Ulbl, J. Vukman, On centralizers of standard operator algebras and semisimple $H^{*}$ algebras, Acta Math. Hungar. 110, 3 (2006), 217-223.

[8] L. Molnár, On centralizers of an $H^{*}$-algebra, Publ. Math. Debrecen 46, 1-2 (1995), 89-95.

[9] J. Vukman, An identity related to centralizers in semiprime rings, Comment. Math. Univ. Carol. 40 (1999), 447-456.

[10] J. Vukman, Centralizers of semiprime rings, Comment. Math. Univ. Carol. 42 (2001), 237-245.

[11] J. Vukman, I. Kosi-Ulbl, An equation related to centralizers in semiprime rings, Glas. Mat. 38, 58 (2003), 253-261.

[12] J. Vukman, I. Kosi-Ulbl, On centralizers of semiprime rings, Aequationes Math. 66 (2003), $277-$ 283.

[13] J. Vukman, I. Kosi-Ulbl, On certain equations satisfied by centralizers in rings, Internat. Math. J. 5 (2004), 437-456.

[14] J. Vukman, I. Kosi-Ulbu, Centralizers on rings and algebras, Bull. Austral. Math. Soc. 71 (2005), $225-234$.

[15] J. Vukman, I. Kosi-Ulbl, A remark on a paper of L. Molnár, Publ. Math. Debrecen. 67 (2005), 419-421.

[16] J. Vukman, I. Kosi-Ulbl, On centralizers of semiprime rings with involution, Studia Sci. Math. Hungar. 43, 1 (2006), 61-67.

[17] J. Vukman, On derivations of algebras with involution, Acta Math. Hungar. 112, 3 (2006), 181-186. 
[18] J. VUKMAN, Identities related to derivations and centralizers on standard operator algebras, Taiwan. J. Math. 11 (2007), 255-265.

[19] J. VUKMAN, M. FošNER, A characterization of two-sided centralizers on prime rings, Taiwan. J. Math. 11, 5 (2007), 1431-1441.

[20] J. Vukman, I. Kosi-UlbL, On two-sided centralizers of rings and algebras, CUBO A Math. Journal 10, 3 (2008), 211-222.

[21] B. ZALAR, On centralizers of semiprime rings, Comment. Math. Univ. Carol. 32 (1991), 609-614. 